\title{
Kinematics-based strategy for the design of a pediatric hand exoskeleton prototype
}

\author{
M. Bianchi, N. Secciani, A. Ridolfi, F. Vannetti, G. Pasquini
}

\begin{abstract}
The number of scientific researches focusing on designing assistive or rehabilitative robotics devices has remarkably increased with respect to just a few decades ago. However, when it comes to developing such devices for children, literature becomes poor. This paper presents the work carried out by the researchers of the Department of Industrial Engineering of the University of Florence in order to design and develop an assistive hand exoskeleton for a child. The results of a preliminary ergonomics test are also given at the end of the paper.
\end{abstract}

\section{Introduction}

An innovative and rapidly growing approach to cope with the remarkable number of pathologies which affect upper and lower limbs is nowadays represented by the robotics rehabilitation and/or assistance [1]. Since the aforementioned pathologies deeply deteriorate the quality of life of the affected people, innovative systems capable of delivering effective high-dosage therapies play a key role in this scenario. Moreover, the fact that most of the robotic rehabilitative and/or assistive devices are meant to be worn for long period by their user focuses the attention on their wearability, which hence becomes a crucial point during the developing phase of such systems. The smaller the compromised limb is, the more challenging becomes designing a wearable exoskeleton. For this and other reasons, only few studies have investigated the exploitation of assistive devices in motor learning for children [2]. The authors have tried to go beyond the limits of the current literature designing an assistive hand exoskeleton for a child with Cerebral Palsy (CP), tailor-made on the user's hand anatomy. The stringent constraints in terms of encumbrance, the difficulties in interacting with the patient and the necessity of developing a system capable of being quickly modified, to follow the growth of the user, have made the whole process a great and stimulating engineering challenge. The whole procedure (visually summarized through the flowchart of Fig. 1), starting from the study and the

\footnotetext{
M. Bianchi, N. Secciani and A. Ridolfi

Department of Industrial Engineering (DIEF), University of Florence,

Via di Santa Marta 3, Florence, Italy

e-mail: matteo.bianchi@unifi.it

F. Vannetti and G. Pasquini

IRCSS Don Carlo Gnocchi, Don Gnocchi Foundation,

Via di Scandicci, 269, Florence, Italy
} 
modeling of the hand anatomy up to the preliminary wearability evaluation, passing through the mechanical design, is described.

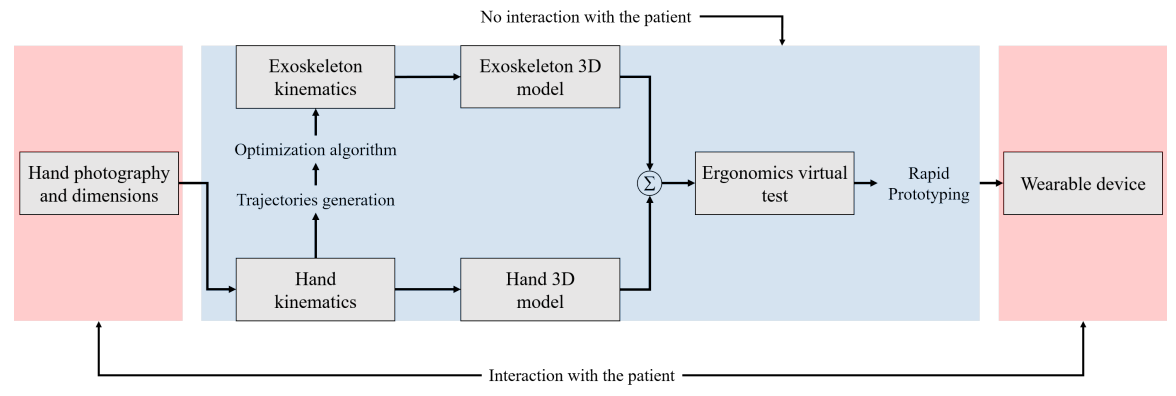

Fig. 1 Model-based design procedure flowchart.

\section{Anthropometric model of the hand}

Interacting with children is not as easy as interfacing with adults. The difficulty to interact with the formers becomes even bigger in a clinical context. With this in mind, the work started from the development of a procedure that would have allowed for studying the patient's hand kinematics with the least possible involvement of the patient himself. Relying only on a few measurements of the limb, a kinematic model of the child's hand, capable of reproducing at best its movements, has been created and used to perform a virtual test of the ergonomics and the functioning of the device before testing it on the real patient.

\subsection{Kinematics}

The kinematic model has been defined to describe the analytical relationship between the angle of each joint of a finger and the fingertip position during virtual grasping gestures, defining a target trajectory the exoskeleton has to pursue.

Considering the hand structural complexity, it is common practice in developing kinematic models to simplify the representation by neglecting those Degrees Of Freedom (DOFs) which do not affect the specific purpose. In the presented kinematic model, based on the finger movement assumptions introduced in [3], these hypotheses are different for the thumb and the long fingers, due to the fact that they present different skeletal structures and consequently show different motion kinds. However, thumb kinematic model has not been considered in this study. Each long finger behaves as a three-link serial planar architecture: three rotational joints with 

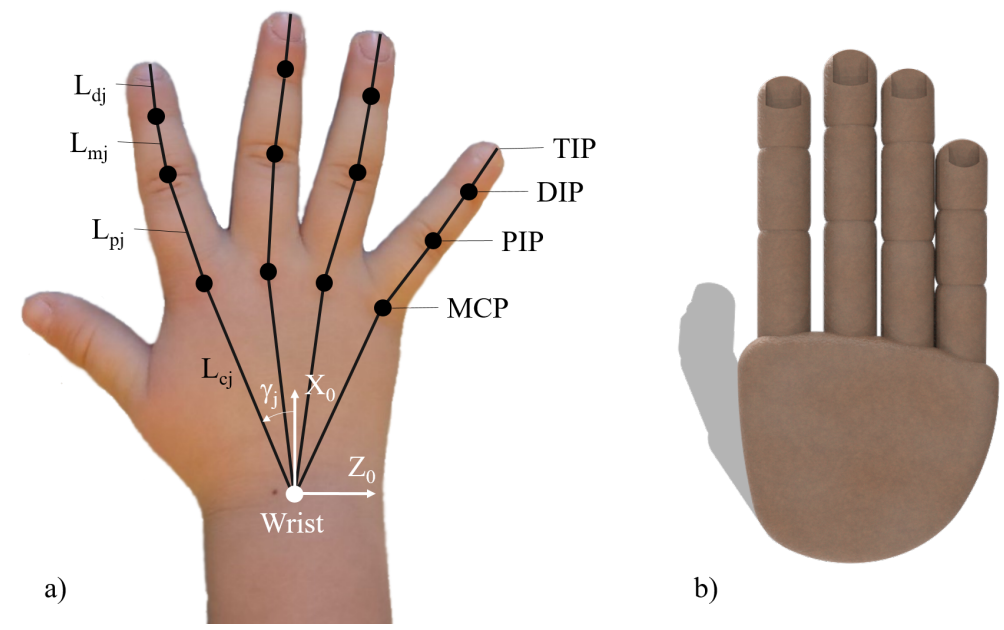

b)

Fig. 2 The kinematic (a) and the 3D (b) models of the child's hand.

parallel axes and moving on the plane perpendicular to the aforementioned axes where the finger longitudinal dimension lies. In fact, even if the human hand model of the long fingers presents $4 \mathrm{DOF}$ each (one flexion-extension movement per joint, and one ab/adduction rotation about the MetaCarpoPhalangeal (MCP) joint axis), in the presented model ab/adduction has not been considered, as the exoskeleton presents a passive DOF, which follows this movement. This will be discussed in Section 3.1. Fig. 2a gives a graphical representation of the kinematic model.

The first step in the model implementation is the specification of a coordinates reference system; the reference system centre is set in the wrist and the axes are oriented so that the $X_{0} Y_{0}$ plane aligns with the sagittal plane, the $X_{0} Z_{0}$ is parallel to the frontal plane and the $Y_{0} Z_{0}$ completes the set laying on the transverse plane. The procedure followed to obtain the complete hand movement uses the Denavit-Hartenberg (DH) convention [4] to identify joints positions and orientations with respect to a wrist coordinate system.

D-H parameters have been defined by exploiting ImageJ open source image processing software which allowed, through a photograph of the hand, to calculate phalanges dimensions. These parameters are collected in Tab 1. Referring to Fig.

\begin{tabular}{ccccc}
\hline Joint & $\theta_{i}$ & $\alpha_{i}$ & $a_{i}$ & $d_{i}$ \\
\hline Wrist & 0 & $-180^{\circ}$ & $L_{c j} \cdot \cos \left(\gamma_{j}\right)$ & $L_{c j} \cdot \sin \left(\gamma_{j}\right)$ \\
MCP & $q_{1}$ & 0 & $L_{p j}$ & 0 \\
PIP & $q_{2}$ & 0 & $L_{m j}$ & 0 \\
DIP & $q_{3}$ & 0 & $L_{d j}$ & 0 \\
\hline
\end{tabular}

Table 1 Long fingers D-H parameters. 
2a, $L_{c j}, L_{p j}, L_{m j}, L_{d j}$ identify, respectively, the Carpal, Proximal, Medial and Distal segments (they are referred to the $\mathrm{j}$-th finger), while $\gamma_{j}$ is the angle between the $\mathrm{j}$-th finger metacarpus and the sagittal plane. PIP, DIP and TIP are, respectively, Proximal and Distal interphalangeal joints and the finger tip.

Once the positions of the fingers joints in the extension configuration have been calculated, flexion trajectories have been obtained by moving the phalanges from the extension to the complete closure configuration. The joint parameters used to identify the two extreme positions (hand fully flexed or fully extended) are reported in Tab. 2. The joints trajectories are defined by a linear variation of these parameters between the aforementioned limits.

The results obtained with this technique have been processed in MATLAB $\mathbb{R}$ environment calculating all the hand joints trajectories and, in particular, extracting the reference trajectory the device has to replicate by exploiting the optimization procedure detailed in [5]. This optimization strategy, taking finger trajectories as targets, allows to automatically define the shape of each finger mechanism which precisely replicates such gestures.

\begin{tabular}{ccc}
\hline & Max Flexion & Max Extension \\
\hline$q_{1}$ & $90^{\circ}$ & $0^{\circ}$ \\
$q_{2}$ & $100^{\circ}$ & $0^{\circ}$ \\
$q_{3}$ & $60^{\circ}$ & $0^{\circ}$ \\
\hline
\end{tabular}

Table 2 Long fingers reference angles for trajectory evaluation.

\section{$2.23 D$ model}

A 3D model of the hand (Fig. 2b) has been developed for testing the coupling between the hand and the exoskeleton. Fingers have been modeled as chains of rigid bodies connected by rotational joints (the kind of joints discussed in Section 2.1 which provide the movement); finger phalanges have then embodied the links modeled by cylinders (with the same radii of the real child's fingers).

\section{Exoskeleton prototype}

The overall architecture of the proposed system originates from the one described in detail in [6]. The main characteristics of the reference exoskeleton are a high customizability (based on a patient-centered approach which implies the acquisition and the reproduction of user's fingers trajectories), wearability and portability, which make it suitable both for performing rehabilitation and providing assistance. Working to adapt the same kind of device to a child's hand, however, has required a 
specific re-design, because of more stringent constraints in terms of available room and bearable weight, and because of the more difficult interaction with the children. As detailed in the following, the developed hand exoskeleton required a two-level customization. The first one (discussed in Sec. 2.1) was represented by the particular kinematics of a children hand. Thanks to an optimization strategy developed by the University of Florence, the same mechanism presented in [6] has been reshaped to fit the child's fingers kinematics. Another important aspect that has been considered was the overall portability of the system (Sec. 3.2): the new device demanded a significant redesign in order not to impede the young user during handling activities.

\subsection{Kinematics}

One of the main contributions provided by the reference device is that its novel 1-DOF mechanism per finger allows to precisely and comfortably reproduce the complex hand kinematics without being forced to use an equally complex robotic device. Dealing with just a single actuated DOF has led to lower size and weight, which have made possible to mount the whole system right on the back of the hand. These assumptions resulted to be essential in designing a device as efficient as simple, thought to be used by a child. The required high adaptability to the user has been reached thanks to the work reported in [6], which presents a detailed explanation of the kinematics of the single-DOF mechanism, and in [5], which describes the Nelder-Mead-based optimization algorithm, used to customize the exoskeleton architecture on the new fingers trajectories. The hand kinematics, returned by the aforementioned model, and the one of the mechanism have been given as inputs to the implemented algorithm resulting in a completely defined geometry of the mechanism, specific for the patient, ready to be imported into a CAD modeling software.

\subsection{Design and manufacturing process}

The hand exoskeleton developed by the University of Florence acts on the four long fingers through a 1-DOF mechanism which actively drives them during the opening movement by using a cable-driven transmission and which passively follows them during closing gesture. As the only one (active) DOF operates in the flexion/extension plane, an additional passive DOF has been added in proximity of each finger MCP joint to make the exoskeleton follow the natural ab/adduction rotation occurring during grasping gestures. This way the active DOF acts on the flexion/extension. In fact, without this passive joint, which restores the alignment between long fingers and exoskeleton mechanisms, the fingers motion is uncomfortable constrained in a single plane, resulting in a reduced mobility.

The whole system dimensions and weight have to be reduced as much as possible leading to an easy-to-handle device even for a child. A single servomotor has been 
exploited and a specific cable driven transmission system has been developed to open all the four long fingers at the same time.

A belt transmission connects the servomotor to a single shaft with four pulleys are spliced to it. Different mechanisms velocities are obtained thanks to the different pulleys diameters, which are calculated depending on users' fingers dimensions. This mechanical architecture allows for a compact and lightweight actuation system.

HS-5495BH High-Torque Servo from Hitec has been chosen to actuate the system. This motor, which has a size of $39.8 \times 19.8 \times 38.0 \mathrm{~mm}$ and weight of $44.5 \mathrm{~g}$, can deliver a maximum torque of $7.5 \mathrm{~kg} / \mathrm{cm}(0.735 \mathrm{Nm})$ at a maximum angular speed of $6.67 \mathrm{rad} / \mathrm{s}$ at $7.4 \mathrm{~V}$.

Finally, children's growth requires to rapidly change the finger mechanisms, producing new geometries properly fitting the continuously changing fingers sizes and gestures. Thus, the mechanical structure has been designed to be 3D-printed in ABS thermoplastic polymer. This choice guarantees, right after periodically measures of the user's hand, to swiftly build the new components placed on the fingers.

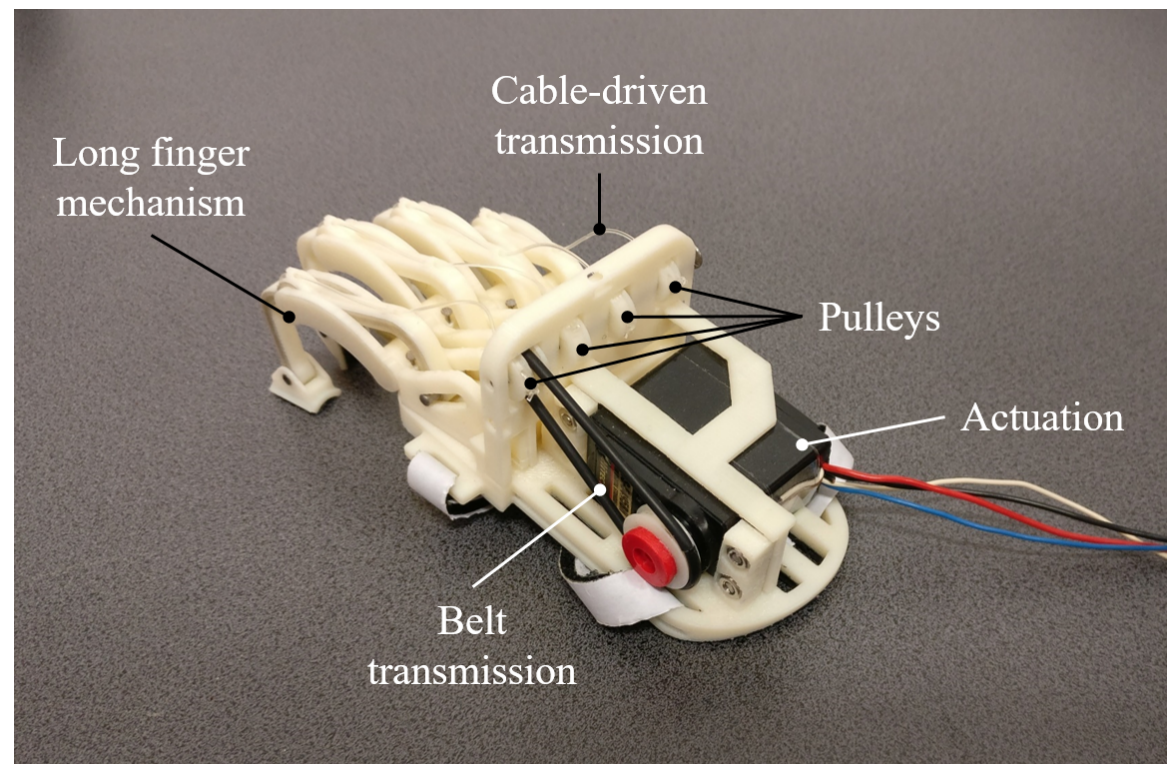

Fig. 3 The hand exoskeleton prototype, ready to be tested. 


\section{Results}

Virtual ergonomic tests have been carried out to assess, even without facing the patient, the hand-exoskeleton coupling and interaction in simple opening gestures. SolidWorks Motion Simulation tools allowed to investigate the overall behavior of the hand when the exoskeleton was worn by the user. Not only the kinematic interaction between the 1-DOF mechanism and the finger has been evaluated, but also the occurrence of interpenetrations between the finger and the device has been checked, verifying mutual coupling during the whole range of motion.

Since the cable-driven actuation system of the exoskeleton is only capable of opening the fingers (the closing gesture is performed by the patient while the servomotor is in charge of controlling the closing velocity), the extension of the hand has been simulated actuating the exoskeleton, while, during the flexion, fingers were modeled to move and the exoskeleton had to follow this kind of gesture. Once the kinematic effectiveness of the device has been tested and the possible interpenetration avoided by modifying the shape of some components, a real prototype has been 3D printed to evaluate the actual wearability of the whole system (Fig. 4). At the time of writing, qualitative tests have confirmed a satisfying adaptability of the device to the child's hand both in fitting size and in following finger trajectories. Up to now, an effective control strategy to actuate the exoskeleton without inconvenience for the young user is under investigation.

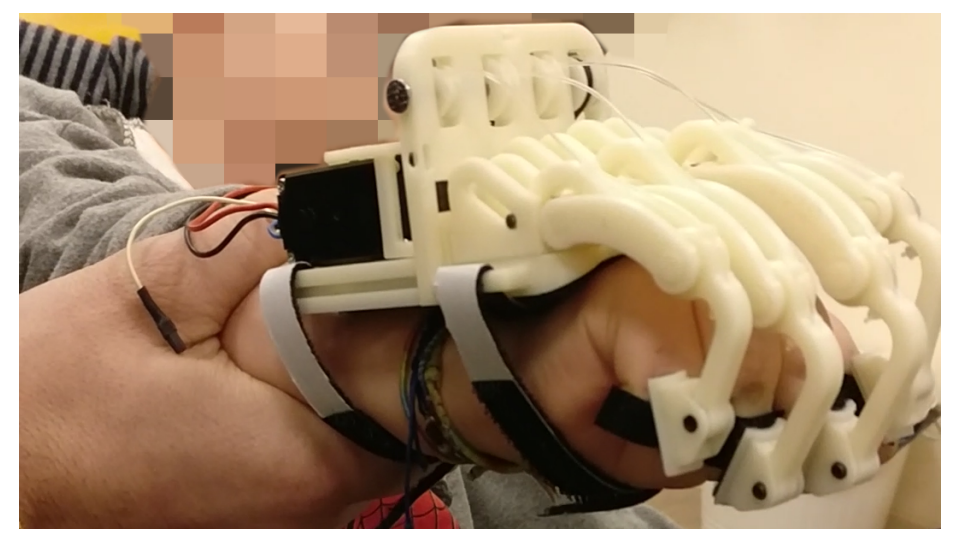

Fig. 4 The hand exoskeleton prototype worn by a child.

\section{Conclusion and further developments}

The small dimensions, the limits of bearable weight, both the high level of safety and customization required, and the difficult interaction make the design of a wear- 
able assistive device for children a real technical challenge, lowering the effective outcome of these systems themselves.

In this paper, a model-based approach allowing the development and production of a hand exoskeleton prototype, tailor-made on a child's anatomy, with just few measurements of the hand, has been presented.

The exploitation of a simple but rapid strategy and technology (as the model-based optimization of the mechanism geometry and the 3D printing process) has allowed to overcome difficulties in interacting with the user and in adapting the device during its fast grow steps, yielding a complete patient-tailored and custom robotic aid. Although the presented work represents a preliminary study, it paves the way to several further developments. As reported in Sec. 4, the first arisen point to be tackled is represented by the necessity of developing specific control and actuation strategies. The current version of the prototype proves that, in fact, the proposed approach leads to a good trade-off between functionality and comfort as concerns the mechanical design and functioning. Nevertheless, a real use (and even usefulness) of the device demands to straightforwardly control the device. The whole interaction between the user and the proposed hand exoskeleton has to be assessed taking into particular account the application on a child. Experimental tests to define the most suitable activation and control strategy in order to evaluate the overall usability of the system are planned.

\section{Acknowledgments}

The authors would like to thank the University of Florence and the Don Carlo Gnocchi foundation which have supported this work.

A special thank goes to Chiara Brogi that has collaborated to the design of the prototype during her Bachelor Thesis.

\section{References}

1. M. Troncossi, M. Mozaffari-Foumashi, and V. Parenti-Castelli, "An original classification of rehabilitation hand exoskeletons," Journal of Robotics and Mechanical Engineering Research, vol. 1, no. 4, pp. 17-29, 2016.

2. J. W. Keller and H. J. van Hedel, "Weight-supported training of the upper extremity in children with cerebral palsy: a motor learning study," Journal of NeuroEngineering and Rehabilitation, vol. 14, no. 1, p. 87, Aug 2017.

3. T. M. W. Burton, R. Vaidyanathan, S. C. Burgess, A. J. Turton, and C. Melhuish, "Development of a parametric kinematic model of the human hand and a novel robotic exoskeleton," in 2011 IEEE International Conference on Rehabilitation Robotics, June 2011, pp. 1-7.

4. B. Siciliano and O. Khatib, Springer handbook of robotics. Springer, 2016.

5. M. Bianchi et al., "An automatic scaling procedure for a wearable and portable hand exoskeleton," in 2016 IEEE 2nd International Forum on Research and Technologies for Society and Industry Leveraging a better tomorrow (RTSI), Sept 2016, pp. 1-5.

6. R. Conti et al., "Kinematic synthesis and testing of a new portable hand exoskeleton," Meccanica, vol. 52, no. 11-12, pp. 2873-2897, 2017. 